\title{
Introduction to the teaching course in Medical Oncology
}

\author{
Ramon Colomer \\ Institut Català d’Oncologia. Girona. Spain.
}

With this issue, we begin the series of topics selected by the SEOM for the Teaching Section of the Revista de Oncología. There are many recent and important changes in the medical treatment of cancer. Furthermore, the specialty of Medical Oncology is developing new intervention strategies for the prevention of cancer and for the palliative therapy of cancer patients. In this Teaching Section series, we have developed 9 chapters dedicated to Medical Oncology that reflect both the changes in therapy and the diversity of approaches to the cancer patient. We will dedicate 3 chapters to the most recent advances in the medical treatment of cancer, focusing in early lung cancer and advanced colorectal cancer, and also in the neoadjuvant treatment of breast cancer. The other 6 chapters will be dedicated to new diagnostic and management tools. We will try to show the outstanding utility of Hospital Cancer Registries for the care of patients with cancer, and for patient management and planning of resources. We will describe the use of tumor banks in genomic and proteomic research, and their future influence in cancer treatment and in the planification of cooperative clinical trials. We will analyse the role of the pharmaceutical industry in cancer research, which is not limited to developing new drugs, but rather to design new endpoints and procedures that help to achieve the cure of cancer. The importance of the diet in the development and progression of cancer will be another of the chapters, and the influence of dietary interventions on the efficacy or the toxicity of chemotherapy will be analysed. Finally, we will deal with two topics to which the Sociedad Española de Oncología Médica (SEOM) has dedicated recently two working groups, a fact which indicates the importance that they have for medical oncologists. The first is related to cancer screening and counseling for individuals that do not have cancer but that are at high risk for developing cancer. The second deals with the new concept of "shared management" of the patient with advanced cancer, by which cancer patients are managed simultaneously by medical oncologists and by palliative care specialist.

We have been very lucky to have a series of well known oncologists and investigators to accept to participate in this Teaching project. I want to thank them all for their time and effort. 